\section{BMJ Open Respiratory Research}

\title{
Clinical characteristics and outcomes of adult patients admitted with COVID-19 in East London: a retrospective cohort analysis
}

\author{
Daryl Cheng (D) , ${ }^{1}$ Claire Calderwood, ${ }^{1,2}$ Erik Skyllberg, ${ }^{1}$ Adam Ainley ${ }^{1}$
}

To cite: Cheng $D$,

Calderwood C, Skyllberg E, et al. Clinical characteristics and outcomes of adult patients admitted with COVID-19 in East London: a retrospective cohort analysis. BMJ Open Resp Res 2021;8:e000813. doi:10.1136/ bmjresp-2020-000813

- Additional material is published online only. To view, please visit the journal online (http://dx.doi.org/10. 1136/bmjresp-2020-000813)

Received 14 November 2020 Revised 14 January 2021 Accepted 16 January 2021

\section{Check for updates}

\section{(C) Author(s) (or their} employer(s)) 2021. Re-use permitted under CC BY-NC. No commercial re-use. See rights and permissions. Published by BMJ.

${ }^{1}$ Respiratory Department, Barking Havering and Redbridge University Hospitals NHS Trust, Romford, UK

${ }^{2}$ Division of Infection and Immunity, University College London, London, UK

Correspondence to Dr Adam Ainley; adam.ainley@nhs.net

\section{ABSTRACT}

Background Descriptions of clinical characteristics of patients hospitalised withCOVID-19, their clinical course and short-term inpatient and outpatient outcomes in deprived urban populations in the UK are still relatively sparse. We describe the epidemiology, clinical course, experience of non-invasive ventilation and intensive care, mortality and short-term sequelae of patients admitted to two large District General Hospitals across a large East London National Health Service Trust during the first wave of the pandemic.

Methods A retrospective analysis was carried out on a cohort of 1946 patients with a clinical or laboratory diagnosis of COVID-19, including descriptive statistics and survival analysis. A more detailed analysis was undertaken of a subset of patients admitted across three respiratory units in the trust.

Results Increasing age, male sex and Asian ethnicity were associated with worse outcomes. Increasing severity of chest X-ray abnormalities trended with mortality. Radiological changes persisted in over $50 \%$ of cases at early follow-up (6 weeks). Ongoing symptoms including hair loss, memory impairment, breathlessness, cough and fatigue were reported in $70 \%$ of survivors, with $39 \%$ of patients unable to return to work due to ongoing symptoms.

Conclusions Understanding the acute clinical features, course of illness and outcomes of COVID-19 will be crucial in understanding the effect of differences in risk, as well as the effectiveness of new interventions and vaccination between the successive waves of the pandemic.

\section{INTRODUCTION}

\section{Background and rationale}

COVID-19 is a respiratory illness caused by the novel coronavirus, SARS-CoV-2. In March 2020, it was designated a pandemic by WHO; at the time of writing there have been more than 20 million cases worldwide and 750000 deaths associated with COVID19. ${ }^{12}$ Within Europe, the UK has experienced a high burden of COVID-19 with more than 300000 cases, the second highest number of total cases in the region and more than 46000 deaths. ${ }^{1}$ During the first surge of the

\section{Key messages}

What were the demographics, clinical characteristics and sequelae for patients admitted to hospital with coronavirus disease 2019 (COVID-19) during the first peak of the pandemic, in a large, urban East London population with high indices of deprivation?

- In our population, increasing age, male sex and Asian ethnicity are associated with increased risk of death, as previously described. Radiological abnormalities and symptoms persist 6 weeks after discharge in over $50 \%$ of patients. Over a third of all currently employed patients who had follow-up had been unable to return to work by their first review.

- This study describes the inpatient experience together with early outpatient outcomes for patients admitted to hospital with COVID-19 during the first wave of the pandemic. It is one of the largest cohorts of patients from two large district general hospitals in London from a deprived urban area, including outcomes from a respiratory clinician-led continuous positive airway pressure unit.

pandemic, London was particularly affected, with the highest age-standardised mortality rate from COVID-19 in the UK (85.7 deaths per 100000 individuals)..$^{3}$

Barking, Havering and Redbridge University National Health Service (NHS) Hospitals Trust (BHRUT) serves one of the largest catchment areas in London for a single NHS trust; the area has been severely affected by COVID-19. The population served is ethnically diverse, with a large Asian cohort, and includes boroughs with high indices of multiple deprivation. ${ }^{45}$

Many groups have described risk factors for severe disease and mortality from COVID19, with non-white ethnicity emerging as a key risk factor, even after adjusting for geographic, socioeconomic, ${ }^{6}$ cardiometabolic and behavioural factors. ${ }^{7}$ The longerterm implications of severe disease remain unclear, however, there is significant concern 
about the potential for prolonged respiratory disability, in part based on data from the previous SARS outbreak. ${ }^{8}$ So-called 'long COVID-19' may however have more wide-ranging consequences, including thromboembolic, neurological and psychological sequelae. ${ }^{9}$

\section{Objectives}

Our first objective was to describe the local cohort of individuals hospitalised with COVID-19 during the first surge (1 March 2020 and 8 June 2020) including inpatient mortality and risk factors for poor outcome. This is in the form of a whole hospital cohort, and nested cohort of patients admitted to respiratory units (RU), for who more detailed data were available. This provides the context in which we established an outpatient follow-up pathway for patients with severe COVID-19.

Our second objective was to describe outcomes for these individuals at six to twelve weeks after discharge.

\section{METHODS}

\section{Design and participants}

This retrospective cohort study included adult patients (age $\geq 18$ years old) admitted across two hospitals that comprise a large NHS Trust in London, UK with a clinical or laboratory diagnosis of COVID-19, from 1 March 2020 to 8 June 2020. The reporting of this study is in accordance with the guidelines set out in the STrengthening the Reporting of OBservational studies in Epidemiology statement.

We present an additional analysis of a nested cohort of patients admitted to the RU. The RUs comprise a total of 90 beds across two sites and admitted the first patients with COVID-19, before rapidly evolving to preferentially admit patients with more severe disease including those requiring non-invasive ventilation (NIV), which was delivered on the unit. Additional data were collected for patients admitted to the RUs between 10 March 2020 (the date of admission of the first patient with COVID19) and 26 April 2020. We describe the demographic, clinical, laboratory and radiological findings and disease course and clinical outcomes of these patients.

As part of development of a local follow-up pathway for COVID-19, we established a prospective cohort of patients invited for postdischarge review, with the primary aim of informing service development. All patients discharged from BHRUT after severe COVID-19 (defined as requiring NIV and/or admission to higherdependency care) are being followed up by this clinic. The first patient invited, and those described here, were those admitted to the RU.

\section{Definitions}

COVID-19 cases were defined as either a laboratory confirmed or clinically suspected diagnosis of COVID19. Patients who tested positive via real-time PCR for SARS-CoV-2 in a respiratory tract sample were classed as laboratory confirmed. A clinically suspected case was defined as a patient who tested negative for COVID19 , but where the attending physician determined that COVID-19 infection was the likely underlying diagnosis, taking into account all clinical, laboratory and radiological data available during the patient's admission, with reference to definitions from Public Health England. ${ }^{10}$

Treatment escalation definitions are defined as: level 1 is for maximal therapy delivered in a medical ward environment; level 2 is for single organ support in a higher dependency area including the RU and level 3 for multiorgan support including invasive mechanical ventilation in an intensive care setting. Ethnicity definitions were those defined by the UK Office for National Statistics. ${ }^{4}$ Obesity is defined as a recorded body mass index of $\geq 30 \mathrm{~kg} / \mathrm{m}^{2}$. Frailty was recorded as the Rockwood Clinical Frailty Score (CFS). An NIV trial is defined as any time on either continuous positive airway pressure (CPAP) or bilevel positive airway pressure. Chest radiograph (CXR) classification and severity was assessed by a member of the respiratory team according to the British Society of Thoracic Imaging (BSTI) classification. ${ }^{11}$

\section{Data collection}

Data were collected from a combination of electronic health records databases, linked using personal identifiers. Data extracted included age, sex, ethnicity, length of stay and discharge outcome. During the period of the study, 'COVID-19 status', as one of 'not clinically suspected', 'suspected COVID-19' or 'confirmed COVID19', were recorded daily as part of the patient flow pathway, which included input from multidisciplinary clinical ward teams during 'board rounds'. For this analysis, the last COVID-19 status prior to discharge was used. We included individuals with 'suspected COVID-19' and 'confirmed COVID-19', defined as above. Additionally, for the nested RU dataset only, these electronic datasets were combined with data collected from manual review of electronic and paper-based patient records. Additional data collected included clinical symptoms and signs, progress during admission including time-updated requirements for organ support and outcome. After record linkage, data were anonymised for analysis.

Follow-up data were collected prospectively for patients attending for follow-up at approximately 6 weeks from their discharge using locally adapted British Thoracic Society guidelines. ${ }^{12}$ Data collected included patient demographics, Medical Research Council (MRC) breathlessness scales at baseline (evaluated retrospectively) and present; results of screening tools for anxiety (Generalised Anxiety Disorder scale-2 (GAD-2)) and depression (Patient Health Questionnaire-2 (PHQ-2)); functional impairment and return to work status. CXRs were reviewed from admission and post-discharge. Patients were informed about clinic appointments prior to discharge. Appointment letters were sent via post and patient pathway coordinators 
attempted to contact all patients by telephone to confirm their appointment. For telephone clinics, two attempts were made to contact patients (one at a scheduled clinic appointment time, and one other in the same half-day clinic). Where patients did not attend face-to-face appointments, clinicians attempted to contact them via phone. Electronic health records were reviewed to determine if the patient had been readmitted to hospital as a reason for non-attendance. If a patient remained out of contact after this, they were offered one further appointment via post, with telephone confirmation.

\section{Analysis}

Analysis was performed using the $\mathrm{R}$ statistical computing environment V.4.0.1. ${ }^{13}{ }^{14}$ We described the study population stratified by inpatient outcome. For continuous variables, analysis of variance tests was used. For categorical variables, Pearson's $\chi^{2}$ tests or Fischer's exact tests were used. Univariable, and then multivariable Cox proportional hazard models were used for survival analyses, with age, gender and ethnicity included as a priori likely confounders. Proportional hazard assumptions were checked using Schoenfeld residuals. For retrospective data, complete case records analysis was used, with missing exposure data assumed to be so at random (there were no missing outcome data). For follow-up data, an intention-to-treat analysis was used, with missing data included in the final analysis.

At the suggestion of a reviewer, we performed a sensitivity analysis to evaluate the impact of our use of a clinical, rather than laboratory confirmed, diagnosis of COVID-19 for our case definition. For this, we repeated the analysis restricting the study population to PCRconfirmed COVID-19 cases only.

\section{Ethics}

These analyses were completed as part of ongoing service evaluation in order to facilitate future planning and ongoing service requirements for patients with COVID-19, in collaboration with the Trust Clinical Audit and Research and Development Team. The Caldicott Guardian was consulted for approval for the use of anonymised patient data.

\section{Patient and public involvement}

Due to the retrospective nature of the study, patients and the public were not involved prior to the collection of data, study design or recruitment to the study. Follow-up data fields were adapted from BTS guidelines, which has patient and public involvement in the form of Lay Trustees. We intend to disseminate the main results of the study using the Trusts' public engagement channels.

\section{RESULTS}

\section{Overall cohort}

\section{Demographics and outcomes}

Data were available for 2091 admissions, representing 1946 patients, with a coded diagnosis of suspected or confirmed COVID-19, admitted from 1 March 2020 to 8 June 2020. Median age was 73 years (IQR 57-84 years); $42.1 \%$ patients were female. Of 1781 patients with ethnicity data available, $1250(70.2 \%)$ were of white ethnicity, 23 (1.3\%) mixed, 313 (17.6\%) Asian, 154 $(8.6 \%)$ black, $41(2.3 \%)$ other. (table 1$)$

There were 594 deaths over 99 days (overall mortality $30.5 \%$; 11.4 deaths per person-year at risk (PY)). There were 414 deaths (12.5 deaths/PY) among admissions to general medical wards and 83 deaths (8.05 deaths/ PY) among admissions to RU. Two hundred and sixteen $(10 \%)$ admissions included a spell in the critical care unit (CCU), with 98 recorded deaths (8.83 deaths/PY). One hundred and seventy-seven patients $(8.5 \%)$ were admitted to CCU without having been transferred from or subsequently to an RU.

The median length of stay was 5 days (IQR 2-11) for all inpatients irrespective of ward. Length of stay was longer where admissions included a spell in CCU (median 13 days (IQR 6-23])) or RU (9 days (IQR 5-15), p<0.001). A considerable tail of individuals had prolonged admissions over 30 days (4\% in general wards, $5 \%$ in the RUs and $19 \%$ in CCU; figure 1). Two hundred and ninetythree admissions included a spell in one of the RUs, of which $248(84.6 \%)$ are included in the subgroup analysis.

\section{Risk factors for inpatient mortality}

Age, gender and ethnicity were included as covariates in the Cox proportional hazards model (table 1). Increasing age was associated with decreased survival probability: compared with patients younger than 60 , patients aged 60-80 had 2.95 times higher rate of death, increasing to 4.44 times in those older than 80 (95\% CI 2.12 to 4.11 and 3.19 to 6.19 , respectively). Men appeared to have a higher mortality rate than women (HR 1.30, 95\% CI 1.09 to 1.56), as did those of Asian ethnicity compared with White (HR $1.40,95 \%$ CI 1.08 to 1.81 ). No difference in survival probability was seen with Black, Mixed or other ethnicity compared with white.

Restricting the study population to PCR-confirmed COVID-19 as a sensitivity analysis, resulted in 1189 admissions, representing 1117 patients. There were 355 deaths over 99 days (overall mortality 31.8\%; 9.3 deaths/PY). There were 257 deaths among admissions to general medical wards (10.5 deaths/PY), 67 deaths among admission to the RU (10.0 deaths/PY), and 75 deaths among admissions to ITU (7.2 deaths/PY). In the Cox proportional hazards model (online supplemental table 1), age, gender and ethnicity were included as covariates. Similar associations to those in the overall population were observed, with increasing age associated with decreased survival probability: patients aged $60-80$ had a 2.7 higher risk of death, rising to 3.9 times higher with patients 
Table 1 Characteristics of patients admitted with PCR confirmed or clinically diagnosed COVID-19 by inpatient outcome, and adjusted HRs from multivariable Cox regression

\begin{tabular}{|c|c|c|c|c|c|}
\hline & Total & Deaths & aHR & $95 \% \mathrm{Cl}$ & $P$ value \\
\hline Overall & 1946 & 594 & - & - & - \\
\hline Age/years $(n=1855)$ & & & & & $<0.001$ \\
\hline Median (IQR) & $73(57-84)$ & $81(72-87)$ & - & - & \\
\hline$<60$ & $560(30.2 \%)$ & $51(9.5 \%)$ & Ref & - & \\
\hline $60-80$ & 666 (35.9\%) & $206(38.3 \%)$ & 3.0 & 2.1 to 4.1 & \\
\hline$>80$ & 629 (33.9\%) & $281(52.2 \%)$ & 4.4 & 3.2 to 6.2 & \\
\hline Gender $(n=1855)$ & & & & & 0.004 \\
\hline Women & $820(44.2 \%)$ & $205(38.1 \%)$ & Ref & - & \\
\hline Men & 1035 (55.8\%) & $333(61.9 \%)$ & 1.3 & 1.1 to 1.6 & \\
\hline Ethnicity $(n=1781)$ & & & & & 0.1 \\
\hline White & 1250 (70.2\%) & $392(76.6 \%)$ & Ref & - & \\
\hline Black & 154 (8.6\%) & $36(7.0 \%)$ & 1.1 & 0.7 to 1.5 & \\
\hline Asian & $313(17.6 \%)$ & 74 (14.5\%) & 1.4 & 1.1 to 1.8 & \\
\hline Mixed & $23(1.3 \%)$ & $5(1.0 \%)$ & 0.8 & 0.3 to 1.9 & \\
\hline Other & $41(2.3 \%)$ & $5(1.0 \%)$ & 0.8 & 0.3 to 1.9 & \\
\hline \multicolumn{6}{|l|}{ Ward $(n=1946)$} \\
\hline General ward & 1500 (77.1\%) & $414(69.7 \%)$ & - & - & - \\
\hline Respiratory unit & $235(12.1 \%)$ & $82(13.8 \%)$ & - & - & - \\
\hline Critical care & 211 (10.8\%) & 98 (16.5\%) & - & - & - \\
\hline
\end{tabular}

aHR, adjusted HRs; ref, reference covariate.

older than 80 years old, compared with those younger than 60. Men had a higher hazard of death, compared with women (HR 1.4, 95\% CI 1.1 to 1.8 ); as did people of Asian ethnicity (HR 1.5, 95\% CI 1.1 to 2.1 ).

\section{RUs and ward-based NIV}

Data were collected from 248 admissions, representing 235 individual patients. One patient was excluded due to highly missing data, and analysis is on 234 patients. Among 28 patients (12\%) with more than one admission recorded, the mean number of admissions was 2.31.

In this nested cohort, the median age was 65 years (IQR 54-80). Most patients admitted to the RU with COVID-19 were men $(66 \%)$. Seventy-six patients died during the data collection period (9.5 deaths/PY) and 20 patients remained inpatient. Patient ethnicity was: 135 (58\%) white, 46 (20\%) Asian, 31 (13\%) black, 4 (2\%) mixed and $18(8 \%)$ other (table 2).

Pre-existing diabetes was present in 69 patients (33\%), and 32 patients were obese (14\%). Among 122 patients who were admitted to the RUs above the age of 65 years, median CFS was 5 (IQR 3-6).

Seventy-nine per cent (186 people) had a treatment escalation plan from a senior clinician. Of those, fifty per cent had a documented clinical decision that treatment escalation to level 3 care was medically appropriate; of
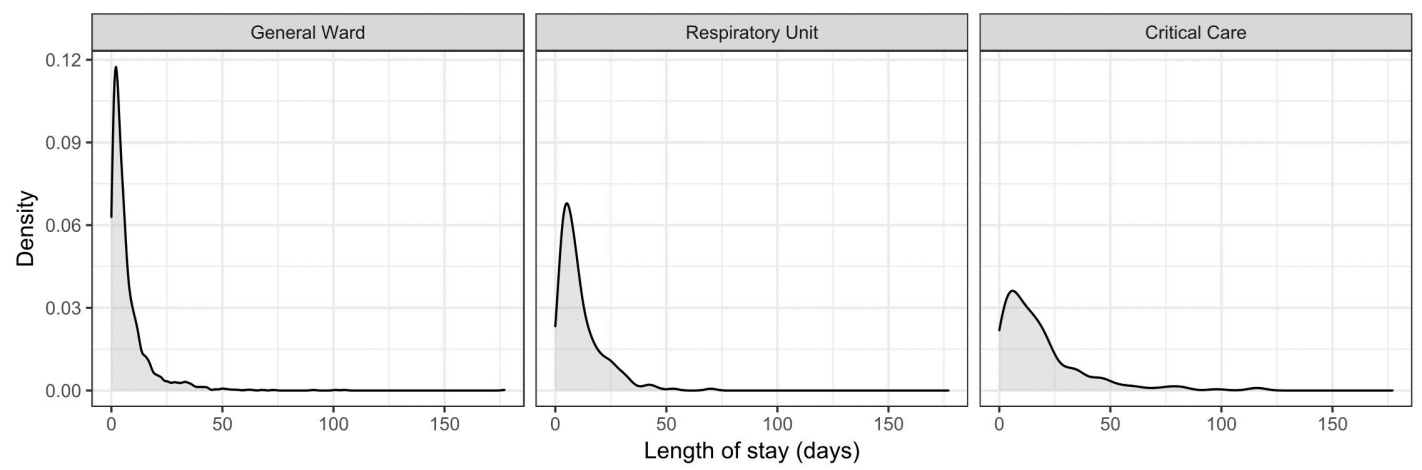

Figure 1 Density plot of patient length of stay by patients admitted to general wards, the RUs and CCU. CCU, critical care unit; $R U$, respiratory unit. 
Table 2 Demographic, clinical and laboratory findings for patients admitted to the respiratory units

\begin{tabular}{|c|c|c|c|c|}
\hline & Total $(\mathrm{N}=234)$ & Died $(\mathrm{N}=76)$ & Alive $(\mathrm{N}=158)$ & $P$ value \\
\hline Age (median, IQR) & 65 (54-80) & $79(70-83)$ & $58(50-71)$ & $<0.001^{*}$ \\
\hline Men & $155(66.2 \%)$ & $48(63.2 \%)$ & $107(67.7 \%)$ & $0.5 \dagger$ \\
\hline Ethnicity & & & & $0.03+$ \\
\hline White & 135 (57.7\%) & 55 (72.4\%) & 80 (50.6\%) & \\
\hline Other & $18(7.7 \%)$ & $3(3.9 \%)$ & $15(9.5 \%)$ & \\
\hline Asian & 46 (19.7\%) & $12(15.8 \%)$ & $34(21.5 \%)$ & \\
\hline Black & $31(13.2 \%)$ & $5(6.6 \%)$ & $26(16.5 \%)$ & \\
\hline Mixed & $4(1.7 \%)$ & $1(1.3 \%)$ & $3(1.9 \%)$ & \\
\hline CFS $(n=76) \neq$ & & & & $0.2 \dagger$ \\
\hline$<5$ & $34(44.7 \%)$ & $13(36.1 \%)$ & $21(52.5 \%)$ & \\
\hline$\geq 5$ & $42(55.3 \%)$ & $23(63.9 \%)$ & $19(47.5 \%)$ & \\
\hline TEP $(n=186)$ & & & & $<0.001 \dagger$ \\
\hline Level 1 & $57(30.6 \%)$ & $33(57.9 \%)$ & $24(18.6 \%)$ & \\
\hline Level 2 & $32(17.2 \%)$ & $17(29.8 \%)$ & 15 (11.6\%) & \\
\hline Level 3 & $97(52.2 \%)$ & $7(12.3 \%)$ & $90(69.8 \%)$ & \\
\hline Pre-existing DM $(n=212)$ & 69 (32.5\%) & $24(36.9 \%)$ & 45 (30.6\%) & $0.4 \dagger$ \\
\hline Obese & $32(13.7 \%)$ & $8(10.5 \%)$ & $24(15.2 \%)$ & $0.3 \dagger$ \\
\hline CRP Adm $(n=219)$ & $127(99)$ & $129(100)$ & $125(99)$ & $0.8^{*}$ \\
\hline Peak $(n=220)$ & $213(286)$ & 267 (479) & $189(115)$ & $0.06^{*}$ \\
\hline$D C(n=200)$ & $97(101)$ & $183(115)$ & $57(62)$ & $<0.001^{*}$ \\
\hline Creat Adm $(n=228)$ & $109(82)$ & $117(98)$ & $106(74)$ & $0.3^{*}$ \\
\hline Peak $(n=225)$ & $148(152)$ & $174(165)$ & $137(145)$ & $0.09^{*}$ \\
\hline$D C(n=204)$ & $110(113)$ & $156(166)$ & $88(68)$ & $<0.001^{*}$ \\
\hline ALT Adm (n=215) & $45(56)$ & $47(71)$ & $44(48)$ & $0.7^{\star}$ \\
\hline Peak $(n=217)$ & $98(253)$ & $146(443)$ & $77(82)$ & $0.06^{*}$ \\
\hline $\mathrm{DC}(\mathrm{n}=187)$ & 66 (192) & $102(338)$ & $50(53)$ & $0.09 \dagger$ \\
\hline Lymph Adm ( $n=233)$ & $1.12(0.97)$ & $1.19(1.47)$ & $1.08(0.62)$ & $0.04 \dagger$ \\
\hline Nadir $(n=225)$ & $0.83(0.92)$ & $0.90(1.50)$ & $0.79(0.46)$ & $0.07 \dagger$ \\
\hline$D C(n=202)$ & $1.31(1.37)$ & $1.18(2.23)$ & $1.37(0.69)$ & $0.04 \dagger$ \\
\hline
\end{tabular}

All tests with Bonferroni correction.

All continuous variables expressed as mean (SD) unless otherwise specified.

*Linear model ANOVA.

†Pearson's $\chi^{2}$ tests.

$\ddagger$ CFS only extracted for patients $>65$ years old.

Adm, admission result; ALT, alanine transferase; ANOVA, analysis of variance; CFS, Clinical Frailty Score; Creat, Creatinine; CRP, C-Reactive

Protein; DC, discharge result; DM, Diabetes Mellitus; Lymph, lymphocyte count; $n$, number of patients with data available; TEP, Treatment

Escalation Plan.

those for whom this was not felt appropriate, $17 \%$ were for NIV (level 2 care) and 33\% for level 1 care. Fortyeight patients had RU-based NIV; this consisted of CPAP in $88 \%$. Mortality was higher in patients who received NIV than those who did not (13.1 vs 8.6 deaths/PY, $\mathrm{p}=0.1$ ).

Of the 27 patients who received NIV, and were considered for admission to the CCU, or did not have a decision that admission to CCU was not medically appropriate, 20 ultimately required admission of which 7 were ultimately intubated. Forty-six per cent $(\mathrm{N}=18 / 35)$ patients receiving NIV in the RU had a trial of awake prone positioning. Awake prone positioning in patients using NIV appeared to be associated with lower mortality (8.2 vs 16.5 deaths/PY, $\mathrm{p}=0.1$ ) however numbers were small. Among patients who required NIV, mortality rate was higher among those for maximal level 2 care compared with those considered appropriate for level 3 care or without a decision for maximal treatment ceiling (34.7 vs 3.0 deaths/PY, $\mathrm{p}<0.0001$ ) (figure 2 ).

\section{Radiological and laboratory findings}

Admission CXRs were classified as normal in $38(17 \%)$, classical for COVID-19 in 121 (53\%), demonstrating 


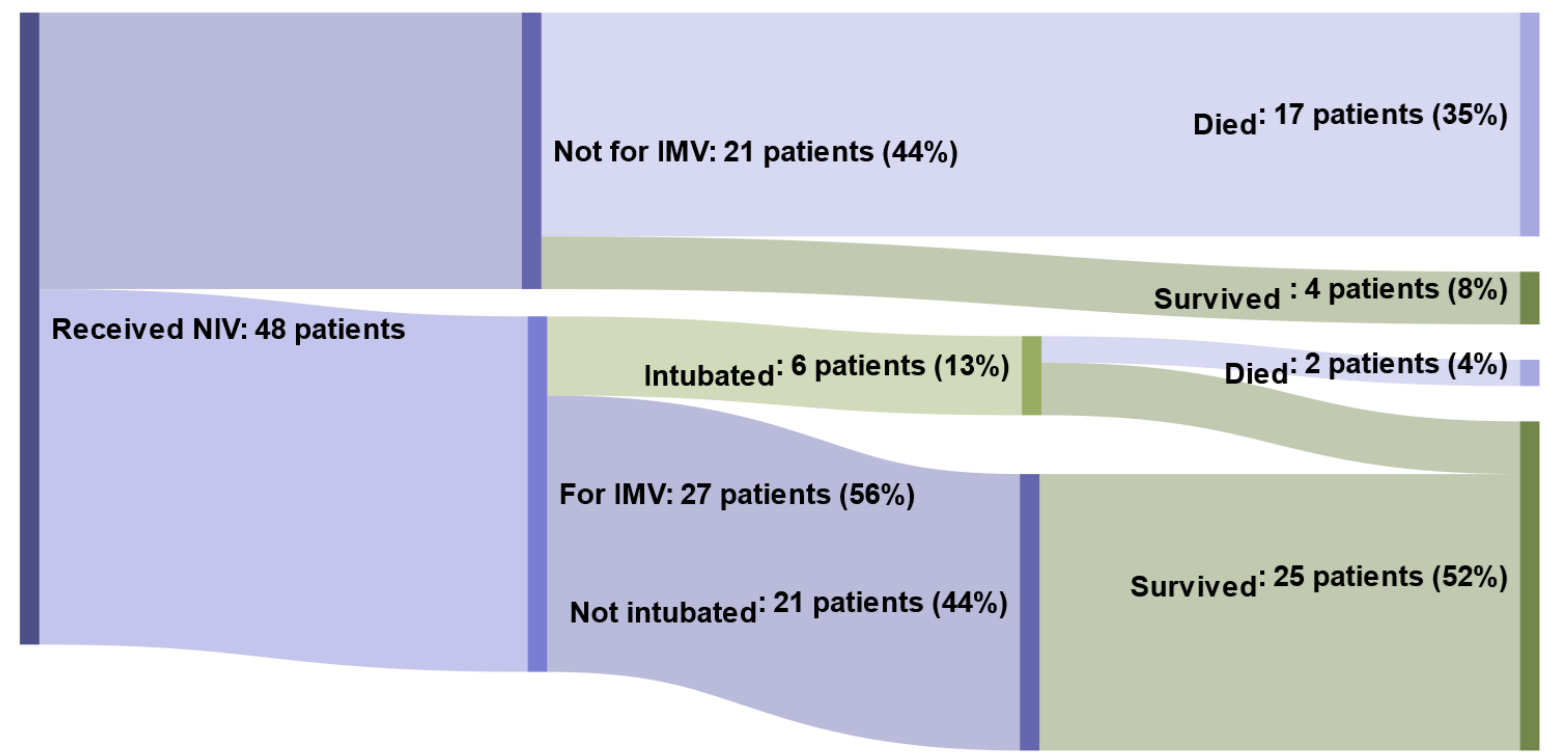

Figure 2 Sankey diagram showing proportion of patients who received non-invasive ventilation (NIV) and outcomes depending on whether Invasive Mechanical Ventilation (IMV) was appropriate and if this was carried out. Percentages refer to total number of patients.

non-COVID-19 features in $46(20 \%)$ and indeterminate in $24(10 \%)$. There was no association between mortality and CXR appearance $(\mathrm{p}=0.4)$. Of the 38 patients who had normal CXR on admission, 26 had further CXRs during their admission, of which 12 were subsequently classed as classical for COVID-19. For patients with admission CXRs classical of COVID-19, mortality increased with degree of radiological severity (Cochran-Armitage, $\mathrm{Z}=-1.9641$, $\mathrm{p}=0.02$ ).

In univariable analyses, there was weak evidence for lower maximum recorded C-reactive protein (CRP) level during admission, lower maximum serum creatinine and lower maximum serum alanine aminotransferase (ALT) among patients who survived to discharge compared with those who died (survivors vs non-survivors, CRP: $189 \mathrm{mg} / \mathrm{L}$ vs $267 \mathrm{mg} / \mathrm{L}$ ( $\mathrm{p}=0.06)$, creatinine: $137 \mu \mathrm{mol} / \mathrm{L}$ vs $174 \mu \mathrm{mol} / \mathrm{L}$ [p=0.09] and ALT: $77 \mathrm{IU} / \mathrm{L}$ vs $146 \mathrm{IU} / \mathrm{L}$ $((p=0.06))$. Analysis of LDH, D-dimer, creatine kinase and ferritin were excluded due to a high degree $(>50 \%)$ of missingness.

\section{Thromboembolic complications}

Eighty-four studies were performed on 66 patients to evaluate for suspected thromboembolic events, including CT pulmonary angiograms (CTPA), CT head and leg Doppler ultrasound scans. Thirteen studies were positive for thrombosis in 11 patients, representing $4.6 \%$ of RU patients. Five patients were diagnosed with pulmonary emboli $(2.1 \%)$, with 5 of 25 CTPAs performed being positive for embolism. Four patients were diagnosed with ischaemic strokes $(1.7 \%)$ and three with lower limb deep vein thromboses (DVT, 1.3\%). One patient had a confirmed lower limb DVT and positive CTPA.

\section{Early outpatient outcomes}

A total of 139 patients discharged from the RU have been offered follow-up at the end of the data collection period. Eight patients $(6 \%)$ required readmission prior to 12-week review (three with pneumonitis, two with pulmonary embolism, two with myocardial infarction and one with stroke). Of the remaining 131, 113 (86\%) attended for review at 6-12 weeks after discharge. Nine patients did not attend despite having two confirmed appointments, one declined due to bereavement, one declined due to existing community COPD follow-up and seven patients did not respond to both appointment letters and telephone calls.

All 113 patients who attended for follow-up had imaging performed; CXR appearances were persistently abnormal in 66 patients $(58 \%)$. Ongoing symptoms were reported in 79 patients (70\%), with fatigue being the most common (69 patients; $61 \%$ ); the median fatigue score was 5 (out of 10; IQR 3.5-7.0). The mean (retrospectively assessed) preadmission MRC breathlessness score was 1.6 (SD 1.05); postdischarge, the mean score was 2.2 (SD 1.2). Forty-one patients $(36 \%)$ had an MRC breathlessness score above their pre-admission baseline at follow-up; this proportion was higher in patients without pre-existing breathlessness ( $45 \%$ vs $22 \%, \mathrm{p}=0.03$ ). Other symptoms included cough $(17 \%)$, memory impairment $(6 \%)$ speech and language issues $(7 \%)$ and hair-loss $(6 \%)$.

Of those who attended for follow-up, $15 \%$ had PHQ-2 scores of $>3 \%$ and $6 \%$ of patients had GAD-2 scores of $>3(6 \%)$, representing a positive screen for depression and anxiety respectively. Of the 69 patients who worked prior to admission, 27 had not yet returned to work at 6 to 12 week review due to their symptoms $(27 / 69=39 \%)$. 


\section{DISCUSSION}

\section{Key findings}

The overall mortality rate in our population was 11.4 deaths/PY, with $22.9 \%$ of admissions requiring a higher level of support than that which could be provided on a general medical ward. Increasing age, male sex and Asian ethnicity identified individuals with higher inpatient mortality rate in adjusted analyses. This is broadly consistent with inpatient cohorts in central London teaching hospitals, national and international data. ${ }^{15-17}$ Increasing CXR severity also trends with mortality.

Our RU nested cohort was, on average, younger than the overall study population, reflecting a selection bias towards patients clinically identified as likely to benefit from more intensive therapy. Men and people of Asian ethnicity are also over-represented, which likely reflects more severe disease among these individuals, necessitating additional support.

In the RU cohort, 11 patients $(4.6 \%)$ had positive studies for thrombosis. The proportion escalated from $\mathrm{CPAP} / \mathrm{NIV}$ to invasive mechanical ventilation, as well as the mortality thereafter, are comparable to data elsewhere. ${ }^{18}$ Few published data exist to describe individual centres' experience of ward based NIV for COVID-19, particularly its use in District General Hospitals with high patient flow.

We report some of the first early follow-up data for patients with severe COVID-19 in the UK. A high proportion of patients remain symptomatic at 6-12 weeks after discharge, particularly with fatigue and breathlessness. These findings are similar to a recently reported central London cohort, ${ }^{9}$ and to a Belgian study, ${ }^{19}$ where a restrictive pattern of lung function was common $(38 \%$, $\mathrm{N}=84 / 101)$. This finding was associated with longer, or critical care admission. It remains unclear to what extent these features reflect COVID-19 specific complications, rather than deconditioning. Our study additionally found that features of mood disorders were common, with elevated anxiety and depression screening scores. Over half of those previously working had not yet returned to work and did not feel ready to do so, concordant with the increasing care needs seen in a significant amount of patients after discharge in other reports. ${ }^{20}$ These findings highlight the importance of holistic assessment and ongoing support following COVID-19 and have led to the inclusion of a clinical psychologist, physiotherapist and occupational therapist within our post-COVID clinic.

\section{Limitations}

The data presented for the whole hospital cohort used electronic databases and record linkage. While coded diagnoses of 'confirmed COVID-19' were checked against laboratory results, there is potential for incorrect coding of 'suspected' cases, resulting in both missed cases, and inclusion of individuals who did not in fact have COVID19. The local process by which these codes were generated (ie, with the clinical team for each individual patient) and use of the last status coded during the admission (when the decision takes into account all available data and the patient's clinical course during the admission) is likely to increase the accuracy of these codes. Additional reassurance may be provided by our sensitivity analysis, which suggests the findings are similar when restricted to PCRconfirmed cases, and the consistency of our results with other cohorts, which used only PCR-confirmed COVID19. Coded diagnoses are usually based on initial diagnosis, and rarely with input from the attending physician, thus the method applied locally provides a rare opportunity to understand the mortality experience of people who were clinically felt to have COVID-19 but who did not have a positive laboratory test.

These analyses were developed as an approach to understand our local experience in the first wave of COVID-19 and inform service requirements for future. The RU cohort represents most unwell COVID-19 patients in our hospitals, outside of critical care and therefore can inform the necessary decisions about optimal resource utilisation during surges of SARS-CoV-2 infection, or other future epidemics. This cohort in fact includes the vast majority of all patients admitted to critical care during the period of the study, however, $8.5 \%$ patients were admitted to critical care but not the RU and are not included. This cohort may not, therefore, be fully representative of all patients needing higher level of care.

For our adjusted estimates of risk factors for mortality, there is potential for unmeasured confounding, given the limited number of variables available from routine data. For example, pre-existing comorbidities are well recognised as risk factors for poorer outcomes from COVID-19, however, these data were not available.

Despite an approach which includes several attempts to contact individuals after discharge, $16(12 \%)$ patients did not attend and had not been readmitted to hospital; their status at 6-12 weeks is unknown. This is similar to the proportion who did not attend face-to-face follow-up in a clinical trial cohort from Bristol. However, in that study, $48 \%$ of those not attending did so due to ongoing shielding. Given the use of telephone appointments, this may be a less common reason in our study; we might suggest therefore that these individuals had fully recovered and felt an appointment was unnecessary or were unable to attend as they had returned to work or care provision. ${ }^{21}$ If so, the missing data for these individuals may, in a complete case records approach, may have led to overestimation of the prevalence of ongoing symptoms and functional impairment. However, we cannot exclude the converse, that patients who did not attend were unable to do so because of breathlessness or other symptoms impacting mobility. As a result, we did not consider it appropriate to assume that non-attenders were asymptomatic.

Finally, COVID-19 is a rapidly evolving situation, both from a public health and clinical perspective with regular new insights into pathogenesis and treatments. In addition, the development of effective vaccines is of 
great promise. These data, from the first wave of the UK pandemic may therefore not be generalisable to the current situation, or that in future.

\section{Interpretation}

These analyses complement the existing literature describing the clinical course and outcomes of hospitalised individuals with COVID-19 in the UK. These data draw from two district general hospitals in a deprived urban location with a large catchment area, very high emergency department demand, and without specialist clinical infectious diseases units. ${ }^{22}$ As a result our study population is larger than previously published individual cohorts ${ }^{23}$ and may be considered more representative of other district general hospitals compared with cohorts from central London. The high deprivation scores among our population should be considered: Barking and Dagenham has the highest index of multiple deprivation (IMD) in London. Other analyses have identified the impact of deprivation on age-standardised mortality rates from COVID-19 in the UK, with a $118 \%$ increase in death rate comparing the least deprived to the most deprived areas, a larger effect than deprivation has on all-cause mortality. ${ }^{3}$ In this context, similar risk factors for mortality were observed.

Increased mortality in individuals of Asian ethnicity has been reported in multiple settings. ${ }^{24}$ We do not find an association between black ethnicity and survival in our cohort, as is reported elsewhere. This difference may reflect low power to detect a difference, given relatively fewer individuals of Black ethnicity compared with white or Asian in our population, or the effect of unmeasured and unadjusted confounding, for example by deprivation indices.

During the COVID-19 pandemic response hospitals have had to undergo rapid review and service redesign in order to manage healthcare demand, particularly ITU capacity. Locally these factors led to an early decision being taken to adapt two existing general medical/ respiratory wards to offer CPAP with high flow oxygen, increased nursing ratios and 24-hour on-site respiratory cover. These analyses were first developed to understand the impact of this service, and we believe they are highly relevant to other clinicians and service managers facing similar decisions. We found that almost $80 \%$ of patients had a documented treatment escalation decision, however there was further improvement possible. NIV/ CPAP was only used for $20 \%$ of admitted patients, with early evidence during the first wave of the UK pandemic demonstrating its effectiveness in reducing mortality. ${ }^{25}$ Three-quarters of individuals receiving NIV and for whom IMV was considered potentially beneficially were ultimately admitted to higher-dependency care, however a minority $(15 \%)$ required intubation. We did not capture the reasons for admission to higher-dependency care, which may include need for renal-replacement therapy and inotropic support as well as deteriorating oxygenation. However, we did also have issues with achieving high-oxygen flow rates through some NIV devices, which anecdotally could also have been a factor. While evaluation of awake prone positioning was not an objective of this study, it was in common use at the time of the study. Given the small numbers and high likelihood of confounding (eg, patients able to tolerate awake prone positioning may be less unwell, be less overweight or have fewer comorbidities than those who are not) it is difficult to draw conclusions from the apparent $50 \%$ reduction in mortality observed with this intervention.

Early reports from Italy and China suggested a high rate of venous thromboembolism in patients with severe COVID-19 pneumonias. ${ }^{26}{ }^{27}$ Data from intensive care units show higher rates of thrombotic complications compared with our respiratory ward cohort. ${ }^{28}$ However, without a non-COVID-19 comparator group it is difficult to interpret whether our data represents a higher rate of thrombosis than would be expected in hospitalised individuals with other viral pneumonias.

Our finding that CXR severity according to BSTI criteria trends with mortality agrees with similar data from the $\mathrm{UK}^{29}$ and CXR scoring systems such as the Italian Brixia score. ${ }^{30}$ Together with studies which correlate semiquantitative CT scores with mortality, ${ }^{31}$ this adds support to the importance of radiological scores in risk stratifying outcome with COVID-19.

The need for more data on recovery from COVID-19 is well recognised. ${ }^{32}$ We report some of the first data on early follow-up outcomes, including describing ongoing symptoms and CXR abnormalities in the majority of patients, with fatigue the most common symptom. The considerable proportion of patients who screened positive for mood disorders, is an important reminder of the need for multidisciplinary input in development of services for individuals recovering from COVID-19, which should include mental health and rehabilitation support. So-called 'long COVID-19' is increasingly recognised, including in patients with milder disease who would not be included in our data. Our findings provide evidence to support development of guidelines for management of postacute COVID-19. ${ }^{33}$ That almost half of patients who had been working prior to their illness felt unable to return to work 6 weeks after discharge also highlights the potential economic impact of COVID-19 for individuals, particularly the more severely affected Black and Minority Ethnic (BAME) and and economically deprived populations.

\section{CONCLUSION}

The UK, and countries around the world, are currently experiencing high numbers of COVID-19 cases, with significant pressure on hospitals and, particularly, intensive care units. While advent of vaccines offers hope, significant time and resource will be required before population coverage is sufficient to impact communitylevel transmission. We describe the inpatient outcomes 
of a large cohort of hospitalised patients with COVID-19, the potential use of ward-based high flow oxygen and CPAP in the management of severe cases, and a large burden of residual symptoms $6-12$ weeks after discharge. These data are contributing to local resource planning and have wider applicability for development of inpatient and outpatient services and evidence-based national guidelines for COVID-19. We again highlight the need for detailed investigation to understand underlying causes of excess mortality in BAME individuals.

Acknowledgements We would like to thank all the respiratory department staff for their invaluable insights which led to the development of these analyses and manuscript, as well as their work throughout the pandemic. We are grateful to our patients, particularly for sharing reflections of their illness which have provided important context, and their families. Particularly, we thank the large team who contributed to data collection: Dr Ana Otamas, Dr Apichaya Amrapala, Dr Ateeb Khan, Dr Charlotte Toms, Dr Daniel Pope, Dr Ebo Dadey, Dr Eshrina Gosal, Dr Gabi Gubo, Dr Grisma Patel, Dr Harish Shankar Kumar, Dr Helen Fielden, Dr Jonathan Ah-Chuen, Dr Kiran Desai, Dr Lani Walshaw, Dr Lennart Graebner, Dr Matthew Birch, Dr Milo Delaney, Dr Natasha 0'Sullivan, Dr Pavan Kumar, Dr Rose Shendi, Dr Shivangee Sinha, Dr Stuart Innes, Dr Tess O'Neill, and Dr Tim Hill.

Contributors All authors contributed to the study design and concept. DC, CC, ES and a wider team in the acknowledgements contributed to data extraction. Data analysis was performed by DC and CC. The manuscript was drafted, revised and reviewed by all authors.

Funding The authors have not declared a specific grant for this research from any funding agency in the public, commercial or not-for-profit sectors.

Competing interests None declared.

Patient consent for publication Not required.

Ethics approval The data collected during this study were reviewed by the Trust Research \& Development and Clinical Audit department review panel and approval was granted without the need for ethical approval as per the NHS Health Research Authority and National Institute for Health Research guidance.

Provenance and peer review Not commissioned; externally peer reviewed.

Data availability statement Data are available on reasonable request. Individual deidentified participant data (including data dictionaries) will be shared. Data that underlie the results reported in this article, after deidentification (text, tables, figures and appendices). Imaging data will not be available to view. Other documents which will be available include study protocol, statistical analysis plan, analytical code. Data will be available beginning from publication and ending five years following article publication. Data will be made available to all researchers who provide a methodologically sound proposal, for their stated aims. Proposals should be directed to daryl.cheng@nhs.net.

Supplemental material This content has been supplied by the author(s). It has not been vetted by BMJ Publishing Group Limited (BMJ) and may not have been peer-reviewed. Any opinions or recommendations discussed are solely those of the author(s) and are not endorsed by BMJ. BMJ disclaims all liability and responsibility arising from any reliance placed on the content. Where the content includes any translated material, BMJ does not warrant the accuracy and reliability of the translations (including but not limited to local regulations, clinical guidelines, terminology, drug names and drug dosages), and is not responsible for any error and/or omissions arising from translation and adaptation or otherwise.

Open access This is an open access article distributed in accordance with the Creative Commons Attribution Non Commercial (CC BY-NC 4.0) license, which permits others to distribute, remix, adapt, build upon this work non-commercially, and license their derivative works on different terms, provided the original work is properly cited, appropriate credit is given, any changes made indicated, and the use is non-commercial. See: http://creativecommons.org/licenses/by-nc/4.0/.

ORCID iD

Daryl Cheng http://orcid.org/0000-0001-9906-4298

\section{REFERENCES}

1 Worldometer. Coronavirus cases.

2 European Centre for Disease Prevention and Control. COVID-19 situation update worldwide, 2020. Available: https://www.ecdc. europa.eu/en/geographical-distribution-2019-ncov-cases [Accessed 14 Aug 2020].

3 Office for National Statistics. Deaths involving COVID-19 by local area and socioeconomic deprivation, 2020. Available: https://www. ons.gov.uk/peoplepopulationandcommunity/birthsdeathsandm arriages/deaths/bulletins/deathsinvolvingcovid19bylocalareasan ddeprivation/deathsoccurringbetween1marchand17april [Accessed 19 May 2020].

4 Office for National Statistics. Ethnicity and National Identity in England and Wales. Available: https://www.ons.gov.uk/peoplepopula tionandcommunity/culturalidentity/ethnicity/articles/ethnicityandnati onalidentityinenglandandwales/2012-12-11\#ethnicity-in-englandand-wales [Accessed 13 Jul 2020].

5 London Datastore. Coronavirus (COVID-19) cases. Available: https:// data.Iondon.gov.uk/dataset/coronavirus--covid-19--cases [Accessed $13 \mathrm{Jul} 2020]$

6 Office for National Statistics. Coronavirus (COVID-19) related deaths by ethnic group, England and Wales. Available: https://www.ons. gov.uk/peoplepopulationandcommunity/birthsdeathsandmarriages/ deaths/articles/coronaviruscovid19relateddeathsbyethnicgroupengl andandwales/2march2020to15may2020 [Accessed 21 Jun 2020].

7 Raisi-Estabragh Z, McCracken C, Bethell MS, et al. Greater risk of severe COVID-19 in Black, Asian and Minority Ethnic populations is not explained by cardiometabolic, socioeconomic or behavioural factors, or by $25(\mathrm{OH})$-vitamin D status: study of 1326 cases from the UK Biobank. J Public Health 2020;42:451-60.

8 Hui DS, Joynt GM, Wong KT, et al. Impact of severe acute respiratory syndrome (SARS) on pulmonary function, functional capacity and quality of life in a cohort of survivors. Thorax 2005;60:401-9.

9 Mandal S, Barnett J, Brill SE. 'Long-COVID': a cross-sectional study of persisting symptoms, biomarker and imaging abnormalities following hospitalisation for COVID-19. Thorax;28.

10 Public Health England. COVID-19: investigation and initial clinical management of possible cases. Available: https://www.gov. uk/government/publications/wuhan-novel-coronavirus-initialinvestigation-of-possible-cases/investigation-and-initial-clinicalmanagement-of-possible-cases-of-wuhan-novel-coronavirus-wncov-infection [Accessed 13 Jul 2020].

11 The British Society of Thoracic Imaging. BSTI COVID-19 guidance for the reporting radiologist. Available: https://www.bsti.org.uk/ standards-clinical-guidelines/clinical-guidelines/bsti-covid-19guidance-for-the-reporting-radiologist/ [Accessed 19 May 2020].

12 British Thoracic Society. British thoracic Society guidance on respiratory follow up of patients with a Clinico-Radiological diagnosis of COVID-19 pneumonia, 2020. Available: https://www. brit-thoracic.org.uk/document-library/quality-improvement/covid-19/ resp-follow-up-guidance-post-covid-pneumonia/

$13 \mathrm{R}$ foundation for statistical computing. $\mathrm{R}$ : a language and environment for statistical computing 2020.

14 Wickham H, Averick M, Bryan J. Welcome to the Tidyverse. J Open Source Softw 2019.

15 Imperial College COVID-19 Response Team. Report 17 - Clinical characteristics and predictors of outcomes of hospitalised patients with COVID-19 in a London NHS Trust: a retrospective cohort studyFaculty of Medicine, Imperial College London. Available: https://www.imperial.ac.uk/mrc-global-infectious-disease-analysis/ covid-19/report-17-clinical/ [Accessed 18 May 2020].

16 Docherty AB, Harrison EM, Green CA, et al. Features of 20133 UK patients in hospital with covid-19 using the ISARIC WHO Clinical Characterisation Protocol: prospective observational cohort study. BMJ 2020;369:m1985.

17 Prado-Galbarro F-J, Sanchez-Piedra C, Gamiño-Arroyo AE, et al Determinants of survival after severe acute respiratory syndrome coronavirus 2 infection in Mexican outpatients and hospitalised patients. Public Health 2020;189:66-72.

18 Singer M. COVID-19 and the role of CPAP. Royal College of physicians. Available: https://player.rcplondon.ac.uk/video/1 n4b2dxmf [Accessed 18 May 2020].

19 Smet J, Stylemans D, Hanon S, et al. Clinical status and lung function 10 weeks after severe SARS-CoV-2 infection. Respir Med 2021;176:106276.

20 Vilches-Moraga A, Price A, Braude P, et al. Increased care at discharge from COVID-19: the association between pre-admission frailty and increased care needs after hospital discharge; a multicentre European observational cohort study. BMC Med 2020;18:408.

21 Arnold DT, Hamilton FW, Milne A, et al. Patient outcomes after hospitalisation with COVID-19 and implications for follow-up: results from a prospective UK cohort. Thorax 2020. doi:10.1136/ thoraxjnl-2020-216086. [Epub ahead of print: 03 Dec 2020]. 
22 London Borough of Barking and Dagenham. Deprivation in Barking and Dagenham. Available: https://www.lbbd.gov.uk/deprivation-inbarking-and-dagenham [Accessed 19 May 2020].

23 Goodall JW, Reed TAN, Ardissino M, et al. Risk factors for severe disease in patients admitted with COVID-19 to a hospital in London, England: a retrospective cohort study. Epidemiol Infect 2020;148:e251.

24 Williamson E, Walker AJ, et al, The OpenSAFELY Collaborative. OpenSAFELY: factors associated with COVID-19-related Hospital death in the linked electronic health records of 17 million adult NHS patients. medRxiv 2020

25 Ashish A, Unsworth A, Martindale J, et al. CPAP management of COVID-19 respiratory failure: a first quantitative analysis from an inpatient service evaluation. BMJ Open Respir Res 2020;7:e000692.

26 Lodigiani $\mathrm{C}$, lapichino G, Carenzo L, et al. Venous and arterial thromboembolic complications in COVID-19 patients admitted to an academic hospital in Milan, Italy. Thromb Res 2020;191:9-14.

27 Cui S, Chen S, Li X, et al. Prevalence of venous thromboembolism in patients with severe novel coronavirus pneumonia. J Thromb Haemost 2020;18:1421-4.
28 Shah A, Donovan K, McHugh A, et al. Thrombotic and haemorrhagic complications in critically ill patients with COVID-19: a multicentre observational study. Crit Care 2020;24:561.

29 Berrill M, Karaj J, Zamfir G, et al. Chest radiographs may assist in predicting the outcome in the early phase of Covid-19. UK district general Hospital experience of Covid-19 first wave. Expert Rev Respir Med 2020:1-5.

30 Borghesi A, Zigliani A, Golemi S, et al. Chest X-ray severity index as a predictor of in-hospital mortality in coronavirus disease 2019: a study of 302 patients from Italy. Int J Infect Dis 2020;96:291-3.

31 Francone M, lafrate F, Masci GM, et al. Chest CT score in COVID-19 patients: correlation with disease severity and short-term prognosis. Eur Radiol 2020;30:6808-17.

32 Lee L, lyer S, Jose RJ, et al. COVID-19 follow-up planning: what will we be missing? ERJ Open Res 2020;6. doi:10.1183/23120541.001982020. [Epub ahead of print: 2605 2020].

33 Greenhalgh T, Knight M, A'Court C, et al. Management of post-acute covid-19 in primary care. BMJ 2020;370:m3026. 